\title{
Le portfolio en éducation des sciences de la santé : un outil d'apprentissage, de développement professionnel et d'évaluation
}

\author{
Porffolio in Health Sciences Education: \\ a Tool for Leaming, Professional Development and Assessment
}

Nicole NACCACHE', Louise SAMSON², Jean JOUQUAN³

\begin{abstract}
Messages clés - Un portfolio est une collection organisée et cumulative de travaux et de réflexions d'un étudiant, qui rassemble des informations sur les compétences quill a développées au cours d'une période plus ou moins longue d'apprentissage. - Le portfolio peut être utilisé comme soutien systématique du processus d'apprentissage et comme source d'information dans le cadre de la démarche évaluative. - L'utilisation du portfolio répond au souci d'aider l'étudiant à développer la composante métacognitive de la compétence professionnelle, en modélisant et en le préparant à une pratique réflexive. $\bullet$ Le contenu et le format d'un portfolio peuvent varier en fonction des buts poursuivis et des contextes d'utilisation. Il comporte : une compilation de documents variés construits à partir des expériences professionnelles rencontrées par l'étudiant; une synthèse articulée des apprentissages développés à partir de ce matériau. - Plusieurs catégories de conditions spécifiques de réussite d'une approche centrée sur le portfolio ont été identifiées : la supervision de l'étudiant par un mentorat adapté; la structure du portfolio et les recommandations formulées aux étudiants; les expériences et le matériel colligé dans le portfolio; le type d'évaluation développée à partir du portfolio. - Un dispositif centré sur le portfolio est potentiellement très fécond mais vulnérable. Les principales menaces identifiées sont: le manque de temps; la mauvaise qualité de la supervision pédagogique ; le manque de clarté des consignes de travail; le caractère non explicite des critères pris en compte pour l'évaluation du portfolio. - L'utilisation du portfolio en tant que support d'évaluation sommative fait l'objet de questions non résolues. Celles-ci concernent : linfluence d'une évaluation sommative sur la nature et la qualité des apprentissages documentés dans le portfolio ; les qualités psychométriques de l'évaluation du portfolio. Des études exploratoires cherchent à opérationnaliser des critères de rigueur alternatifs issus de la recherche qualitative.
\end{abstract}

Mots clés Portfolio ; pratique réflexive ; apprentissage ; évaluation formative ; évaluation sommative ; développement professionnel

Key Messages $\bullet$ A portfolio is an organized collection of material and reflections given by a student, which records information based on the development of his competences and performances over a certain period of training. $\bullet$ A Portfolio can be used as a systematic tool to support learning (portfolio-based learning) as well as a source of information to assess (portfolio-based assessment). - Using a portfolio allows the student to develop the metacognitive component of a professional aptitude, while conditioning him to a reflexive practice. - The content and the format of a portfolio can vary according to the objectives and contexts of use. It comprises: 1) an assorted collection of material and evidence built from students' professional experiences, 2) a reflective overview that summarizes the learning from these experiences. - The successful reflective use of portfolios must take into account the following conditions: adapted coaching; appropriate portfolio structure and guidelines; relevant experiences and material; development of a proper assessment based upon the portfolio. Portfolios are potentially valuable instruments for the development and the assessment of a reflective practice. However, they present some weaknesses such as: a lack of time; a deficiency of mentoring and feedback; indefinite guidelines; an unclear description of assessment criteria for the portfolio. - The use of a portfolio as a tool for summative evaluation is still under debates. Unanswered questions concern: 1) the influence of a summative evaluation on the nature and the quality of learning and 2) psychometric attributes of the portfolio assessment. Research is currently in progress to investigate and validate the use of other assessment criteria derived from qualitative research instead of psychometric studies.

Key words Portfolio; portfolio-based learning; formative assessment; summative assessment; professional development.

Pédagogie Médicale 2006;7:110-27 


\section{Introduction}

Depuis plusieurs années, une littérature abondante en éducation médicale est consacrée au portfolio ${ }^{1,2}$. Utilisé depuis longtemps dans le milieu de la formation artistique, où il désignait les travaux les meilleurs et les plus représentatifs sélectionnés par l'étudiant pour rendre compte de son savoir-faire, il s'est développé progressivement dans les différents milieux de l'éducation générale (enseignement primaire, secondaire, collégial ou universitaire) à partir des années $85^{3}$. En formation professionnelle, il a d'abord été utilisé dans les domaines qui partageaient certaines similitudes avec la formation artistique, comme par exemple la formation en architecture. Dans le champ de l'éducation en sciences de la santé, il a commencé à être employé à une large échelle au RoyaumeUni, initialement dans le contexte de programmes de formation d'infirmières ${ }^{4}$ et de sages femmes ${ }^{5}$ puis, notamment, de programmes de formation en médecine générale $^{6}$. Il fait aujourd'hui l'objet, de par le monde, de développements exploratoires ou achevés, plus ou moins aboutis, dans de multiples contextes de formation de professionnels de santé ${ }^{1}$.

Le terme portfolio désigne d'abord un dossier (portefeuille) permettant une compilation concrète de données de nature diverse, produite par un étudiant pour rendre compte de son travail et illustrer ses efforts, ses progrès et ses réalisations dans un ou plusieurs domaines. Au-delà de sa réalité matérielle, en tant qu'outil, ce dossier peut être dédié au soutien systématique du processus d'apprentissage (portfolio-based learning) ${ }^{\prime}$; un tel usage est surtout développé au sein des programmes de formation qui se réclament du paradigme d'apprentissage $^{7}$, c'est-à-dire ceux qui privilégient le rôle de l'étudiant qui apprend en traitant activement des informations, en interaction avec ses pairs et ses professeurs, à partir des problèmes qu'il doit résoudre ou des projets qu'il doit développer. En tant que document contenant des informations et des traces objectives renseignant sur les apprentissages réalisés, le portfolio peut aussi servir de base à la démarche évaluative (portfolio-based assessment) ${ }^{l}$; une telle utilisation est notamment associée aux dispositifs de formation qui privilégient l'enchâssement de l'évaluation dans les situations d'apprentissage, tels que ceux qui s'inscrivent dans les courants de l'évaluation authentique (authentic assessment) ou de l'évaluation des performances (performance assessment $)^{8}$.

La contrepartie de cette popularité croissante et de ces multiples usages est que l'on utilise parfois le même terme pour désigner des intentions, des instruments, des formats d'intervention et des pratiques pédagogiques relativement hétérogènes ${ }^{9}$, s'adossant à des cadres conceptuels pas toujours très bien stabilisés, ni même explicites. Le risque existe que soient entretenues des confusions à l'égard des multiples approches du portfolio. Les expériences d'utilisation du portfolio en éducation des sciences de la santé n'échappent pas à ces ambiguïtés. En outre, bien que la fécondité potentielle du portfolio par rapport à la démarche d'apprentissage dans le cadre d'un développement de compétences professionnelles soit globalement reconnue $^{10,11}$, les impacts réels du portfolio sont encore incomplètement documentés en éducation des sciences de la santé $^{12}$. Des questions quant à la place du portfolio dans le cadre de la démarche évaluative sont par ailleurs encore non résolues et font l'objet de débats ${ }^{7,13}$. La présente contribution se propose d'apporter une synthèse didactique provisoire des bases conceptuelles, des finalités, des applications opérationnelles, des forces et des limites de l'usage du portfolio en éducation des sciences de la santé et notamment en formation médicale initiale.

\section{Définitions}

Beaucoup de chercheurs ont proposé leur propre définition du portfolio. Les différents énoncés rendent compte des divers positionnements conceptuels adoptés et des différentes finalités assignées au portfolio, respectivement, par les uns et par les autres.

La plupart des définitions originales formulées en langue française ne se rapportent pas spécifiquement aux usages du portfolio en enseignement supérieur ni, a fortiori, à ses transpositions en éducation des sciences de la santé.

Pour Legendre, dans son Dictionnaire actuel de l'éducation $^{14}$, le portfolio est un " document écrit dans lequel les acquis de formation d'une personne sont définis, démontrés

1- Département d'Anesthésie-Réanimation - Hôpital Hôtel Dieu de France (affilié à la faculté de médecine de l'Université Saint Joseph de Beyrouth) - Beyrouth - Liban

2- URDESS (Unité de recherche et de développement en éducation des sciences de la santé) Faculté de médecine - Université de Montréal - Canada

3- Bureau de pédagogie médicale - Faculté de médecine de l'Université de Bretagne Occidental - Brest - France

Correspondance : Nicole Naccache - Hôtel Dieu de France - rue Adib Ishaac - BP 166830 - Beyrouth - Liban - Tél. : +9611615 300 Télécopie : +9611615 295 - Mailto:drnaccache@yahoo.fr 
et articulés en fonction d'un but ». Ainsi défini, il constitue un dossier de présentation qui, dans une certaine mesure, se rapproche du curriculum vitae, à la réserve près que le portfolio contient un élément de démonstration d'actions réalisées dans un but précis, qui n’est habituellement pas présent dans un curriculum vitae ${ }^{14,15}$.

Forgette-Giroux et Simon ${ }^{16}$ mettent davantage en exergue la dimension pédagogique du portfolio, défini comme « [...] un recueil continu et systématique d'une variété de données qui témoignent du progrès de l'élève par rapport à la maîtrise d'une compétence jugée à partir d'une échelle descriptive».

Les définitions qui se réferent à l'usage du portfolio en éducation des sciences de la santé ne font pas l'objet d'un consensus. Plusieurs points de vue récents illustrent ces divergences qui témoignent de la variété des usages du portfolio et des contextes d'utilisation. Cole ${ }^{17}$ plaide pour une définition générique minimaliste du portfolio, conçu comme une « collection de données factuelles (evidence), développée et présentée pour un but spécifique ». Rees ${ }^{18},{ }^{19}$ considère que cela rend mal compte de la valeur ajoutée attendue du portfolio lorsqu'il est employé en éducation médicale et souligne qu'il est important de distinguer le portfolio d'autres outils moins ambitieux, tel que le simple carnet de bord (logbook). Pour la plupart ${ }^{1,2}$, au minimum, un portfolio doit être une " collection organisée et cumulative de travaux et de réflexions d'un étudiant, qui rassemble des informations sur les compétences qu'il a développées au cours d'une période plus ou moins longue d'apprentissage ». Driessen et al. ${ }^{13}$ placent les notions de processus d'apprentissage et de niveau de compétence au centre de ce dont un portfolio doit rendre compte. Une différence d'appréciation notable concerne la place et l'importance de la composante "réflexive " dans le portfolio ${ }^{17}, 19$. Dans le cadre de la présente contribution et en accord avec plusieurs auteurs ${ }^{2,19}$, nous considérerons que, lorsque le portfolio est employé dans le cadre d'un dispositif centré sur le développement de l'expertise professionnelle en santé, la dimension réflexive est primordiale. Un autre point de discussion concerne l'utilisation du portfolio comme outil d'évaluation des apprentissages. Sa légitimité en tant que support d'évaluation formative n'est guère discutable dans la mesure où il a été précisément introduit en éducation médicale pour permettre une évaluation des performances en contexte authentique ${ }^{2,6,13}$. En revanche, les conditions dans lesquelles il peut être employé aux fins d'une évaluation sommative font l'objet de discussions ${ }^{20}$.

Au total, il semble raisonnable d'admettre avec plusieurs auteurs qu'un portfolio utilisé dans le cadre du développe- ment de l'expertise professionnelle en sciences de la santé doit nécessairement intégrer trois composantes, en lien avec les tâches professionnelles authentiques développées par les étudiants : un support à la planification des apprentissages, un support à l'évaluation formative, un support à la démarche réflexive ${ }^{2,19,21}$.

Nous argumenterons cette conception dans la section consacrée aux bases conceptuelles du portfolio et nous discuterons plus loin les problèmes qu'elle peut poser, notamment si l'on souhaite utiliser le portfolio à des fins d'évaluation sommative.

\section{Les bases conceptuelles de l'utilisation du portfolio en éducation médicale}

Lintroduction du portfolio dans les dispositifs de formation des professionnels de santé résulte de développements éducationnels et de pratiques pédagogiques qui puisent leurs fondements au sein de plusieurs cadres conceptuels. Nous en évoquerons certains en les regroupant sous deux chapitres concernant respectivement les concepts de pensée, de pratique et d'apprentissage réflexifs d'une part, les travaux concernant les caractéristiques souhaitables pour les dispositifs curriculaires dédiés au développement de l'expertise des professionnels de santé, d'autre part.

\section{Des conceptions convergentes de la pensée réflexive, de la pratique réflexive et de l'apprentissage réflexif}

Le concept de pensée réflexive a été développé initialement par John Dewey ${ }^{22}$, pédagogue américain dont l'œuvre a considérablement influencé la vie scolaire des EtatsUnis et la pensée pédagogique de nombreux pays dans la première moitié $\mathrm{du} \mathrm{XX}^{\mathrm{e}}$ siècle. Pour lui, la pensée réflexive -assimilable à la notion de pensée critique ${ }^{23}$ - est provoquée par un évènement qui induit un doute, une perplexité ou une incertitude et qui conduit un individu à rechercher une explication et des solutions. Il décrit cinq étapes dans l'organisation opérationnelle d'une telle expérience : la reconnaissance d'un problème ; la définition de ce problème ; la formulation de solutions hypothétiques ; l'anticipation des résultats possibles et la mise à l'épreuve des hypothèses résolutives.

On doit cependant à Schön ${ }^{24,25}$ d'avoir assuré une fortune beaucoup large à ce champ conceptuel en ayant construit, à partir de ses observations de plusieurs professions, le concept de praticien réflexif et d'avoir proposé des pistes pour guider la formation de tels professionnels. Selon lui, les pratiques professionnelles ne se limitent pas à l'usage d'un savoir préexistant mais elles créent aussi des savoirs 
de novo. Le concept de pratique réflexive s'appuie notamment sur deux notions. La première est que toute action professionnelle complexe résulte d'un jugement et d'un processus de décision correspondant à une réflexion dans (c'est-à-dire pendant et à propos de) l'action; aucune d'entre elles n'est le produit d'un pur automatisme. La seconde est que les connaissances et les compétences sur lesquelles s'appuient cette réflexion dans l'action ne sont pas exclusivement issues de savoirs savants codifiés, tels qu'ils sont produits par exemple par la recherche scientifique ; elles prennent aussi en compte un savoir tacite, expérientiel, construit par les praticiens eux-mêmes à partir d'une réflexion développée $a$ posteriori sur leurs actions et qu'ils réutilisent dans leurs actions ultérieures. Il s'agit d'une autre manière de concevoir l'articulation entre la " théorie » et la "pratique » : la séquence ne se fait pas de façon unidirectionnelle de la théorie vers la pratique mais, grâce à une activité mentale de haut niveau, les deux domaines se nourrissent mutuellement.

Le concept de pratique réflexive est fortement cohérent avec la perspective du traitement de l'information appliquée au développement de l'expertise professionnelle ${ }^{26}$. Ericsson insiste sur le fait que l'expertise professionnelle -considérée comme étant le niveau le plus abouti de la compétence professionnelle- ne peut être maintenue et développée qu'au prix d'une pratique particulière, consciente et volontariste, explicitement orientée vers l'apprentissage, et qu'il désigne sous le terme de pratique intentionnelle (deliberate practice) ${ }^{27}$; il s'agit en quelque sorte de s'opposer systématiquement à la facilité que procure l'automatisation graduelle des performances, en exploitant des situations professionnelles privilégiées pour développer des apprentissages visant un niveau encore accru de compétences. Sans une telle attitude, la simple accumulation d'expériences ne permet pas de construire puis, lorsqu'elle est acquise, de développer ni même de maintenir l'expertise. Concrètement, Mamede et Schmidt ${ }^{28}$ illustrent cette notion en évoquant qu'un médecin généraliste qui développerait de façon explicite des apprentissages en profondeur, à partir de la prise en charge suggérée par un médecin spécialiste à qui il a référé un patient qui lui posait problème, dans le but d'améliorer ses pratiques ultérieures face à des patients présentant des problèmes de santé similaires, s'inscrirait dans le cadre d'une telle pratique intentionnelle. Pour eux, les caractéristiques de la pratique intentionnelle sont : une réflexion critique sur ses pratiques, l'attention portée aux faiblesses ou aux limites de ses performances, notamment lorsque l'on est confronté à une difficulté ou un problème inattendu, avec l'intention d'améliorer sa performance. Ils considèrent que, définie de cette manière, la locution « pra- tique intentionnelle » est synonyme de "pratique réflexive». On utilise parfois le terme générique d'apprentissage réflexif pour désigner les apprentissages, à la fois en tant que processus et que produits, qui sont effectués dans le cadre d'une telle démarche de réflexion dans et sur l'action ${ }^{29}$.

King et Kitchener ${ }^{30}$ décrivent pour leur part trois niveaux qui rendent compte du développement d'une pensée critique ou réflexive : la pensée pré-réflexive, la pensée quasiréflexive et la pensée réflexive. Ils les associent à la capacité d'avoir une vision de moins en moins réductrice des savoirs codifiés et d'appréhender de mieux en mieux la complexité et l'incertitude. Au stade de pensée réflexive, la connaissance est perçue comme le résultat d'un processus de recherche reconnu, grâce auquel des solutions acceptables à des problèmes mal structurés sont construites. La pertinence de ces solutions est évaluée en termes de probabilité ou de vraisemblance, compte tenu de l'état de la question, et sera ré-évaluée si de nouvelles données probantes ou de nouveaux outils deviennent disponibles. Les convictions sont justifiées de façon probabiliste sur la base d'une démarche interprétative qui intègre les données probantes, le risque de conclusions erronées et une appréciation des conséquences prévisibles de décisions alternatives. Dès lors, les décisions sont argumentées de façon dialectique et justifiées comme étant, à un instant donné, le meilleur compromis compte tenu des données disponibles.

Pris dans leur ensemble, ces repères conceptuels expriment l'idée qu'il n'y a pas d'expertise professionnelle sans développement de fortes capacités métacognitives. Cette notion désigne la faculté pour un individu de prendre conscience de son propre fonctionnement intellectuel et, par exemple, de porter un jugement, pendant l'action, à l'égard de la qualité et de la pertinence des décisions prises et de leurs résultats.

Les approches qui recourent au portfolio sont l'une des réponses pédagogiques possibles à la nécessité pédagogique d'aider les futurs professionnels à développer cette composante métacognitive de la compétence professionnelle $^{1,2,30,31}$. Pour Parboosingh ${ }^{32}$, elles sont en outre cohérentes avec la perspective du professionnalisme qui renvoie à une conception dynamique de la pratique professionnelle, qui prend en compte les attentes de la société en subordonnant l'intérêt du médecin à celui du patient et qui conduit le professionnel à développer des réflexions sur ses actions et ses décisions dans le but d'améliorer ses connaissances tout en étant critique sur sa pratique quotidienne ${ }^{33}$. C'est une manière de faire face à l'incertitude et à la complexité qui caractérisent les problèmes de santé, en inscrivant les pratiques professionnelles dans 
le cadre d'une réflexion éthique et morale, qui intègre les valeurs humanistes d'honnêteté, d'intégrité, de compassion et de respect des autres.

\section{Des conceptions convergentes concernant les curriculums dédiés au developpement de l'expertise des professionnels en sciences de la santé}

Depuis près d'une vingtaine d'années, une réflexion critique s'est développée à l'égard des programmes de formation des professionnels de santé et en particulier des futurs médecins ${ }^{1,2}$. De nombreux organismes ont élaboré des recommandations qui s'appuient sur une description des caractéristiques attendues chez les futurs diplômés. Prises globalement, ces recommandations stipulent que les curriculums dédiés à la formation des professionnels de santé doivent être définis à partir du répertoire des compétences professionnelles visées, ce dont rendent compte les concepts de competency-based education ${ }^{34}$ ou outcomebased education ${ }^{35}$. En cohérence avec ces énoncés, ces recommandations stipulent également que les procédures d'évaluation doivent s'appuyer sur des dispositifs permettant de documenter l'acquisition effective de ces compétences, en tant que résultats des apprentissages ad hoc visés par de tels curriculums, ce que traduit la notion d'outcome-based assessment ${ }^{36,37}$.

Dans un contexte européen, au Royaume-Uni, le General Medical Council a publié en 1993 le rapport Tomorrow's doctors qui a été réactualisé en $2003^{38}$. Il stipule que les programmes de formation initiale doivent avoir comme résultat explicite de former des cliniciens dont les pratiques satisfont les standards de qualité, capables de maintenir leur compétence professionnelle, de communiquer adéquatement avec leurs patients, de travailler en collaboration avec leurs pairs et les autres professionnels de santé, et démontrer des caractéristiques de probité.

Elaboré en 1996 dans un contexte nord-américain, le projet du Collège royal des médecins et chirurgiens du Canada, connu sous la dénomination de Projet CanMEDS, réactualisé en $2005^{39}$ et dont le sous-titre de sa version initiale fait clairement référence à des attentes sociétales ${ }^{40}$, est très convergent avec ce rapport. Il énonce que les compétences clés du médecin doivent être au service de sept rôles professionnels : expert clinicien, communicateur, collaborateur, gestionnaire, promoteur de la santé, érudit et professionnel.

Pour faciliter l'opérationnalisation de tels objectifs, plusieurs équipes, comme par exemple celle de Harden $e t$ al. . $3,36,41$ à Dundee, ont élaboré un modèle didactique de la compétence médicale qui en répartit 12 composantes en trois niveaux concentriques. Le noyau central représente ce que le médecin compétent est capable de faire (doing the right thing) et fait référence à sept domaines de tâches élémentaires (task performance): examen clinique, gestes techniques, investigations complémentaires, prise en charge globale du patient, prévention et promotion de la santé, communication, traitement de l'information médicale et scientifique. La couche médiane représente comment il appréhende sa pratique (doing the thing rigth) et fait référence à trois dimensions de la compétence médicale (approach to tasks): compréhension des bases scientifiques bio-cliniques et sociales de la santé, compréhension des bases éthiques de l'action médicale, exercice judicieux des processus de raisonnement clinique et de prise de décision. Enfin, la couche supérieure fait référence au médecin agissant en tant que professionnel (the right person doing it), ce qui implique qu'il réfléchisse à sa place dans le système de santé et qu'il acquière des capacités de développement personnel.

Tant les recommandations élaborées par les organismes professionnels que les différents guides développés autour du concept d'outcome-based medical education, tels que ceux diffusés par l'équipe de Dundee, mettent en exergue la dimension métacognitive et réflexive des caractéristiques visées chez les professionnels de santé. Aux yeux de leurs promoteurs, il s'agit d'une condition essentielle pour garantir des standards d'excellence de pratique médicale. Le rapport CanMEDS ${ }^{39}$ insiste explicitement sur « [...] certaines aptitudes essentielles comme l'apprentissage continu, l'auto-évaluation, $[. .$.$] » et indique que « les$ méthodes d'apprentissage doivent refléter aussi fidèlement que possible l'exercice réel de la médecine (apprentissage contextuel) ». Il rappelle que "l'apprentissage expérientiel se veut un mode d'apprentissage essentiel et efficace en résidence ". Le document Tomorrow's doctors ${ }^{38}$, pour sa part, énonce dès la première recommandation ayant trait au contenu curriculaire que les étudiants " doivent disposer de temps dédié à la réflexion et à leur développement personnel "; il indique que " les étudiants doivent avoir un contrôle plus grand de leur propre apprentissage et développer des habiletés d'apprentissage auto-dirigé » et prévoit explicitement qu'ils « doivent avoir des occasions de développer de telles activités » et " [...] présenter les résultats de leur travail verbalement, $[\ldots]$ ou par écrit ».

L'approche que recouvre les locutions "portfolio-based learning " et "portfolio-based assessment ", ${ }^{111}$ est une réponse à ces préoccupations. Elle propose le portfolio comme support d'un apprentissage qualifié de réflexif, en référence explicite à la pratique réflexive. Elle fait l'hypothèse que le portfolio peut induire un processus favorisant l'évolution vers une culture pédagogique " centrée sur 
l'apprenant ${ }^{42,43,44}$. Le portfolio vise à développer l'autonomie du futur professionnel dans sa démarche d'identification de ses besoins. Il l'encourage à planifier ses activités d'apprentissage à partir des tâches professionnelles authentiques auxquelles il est exposé et à s'auto-évaluer, dans une démarche de pratique critique et réflexive. Pour autant, l'approche du portfolio based learning ne postule pas que l'autonomie de l'apprenant soit d'emblée acquise et elle reconnait la nécessité que celui-ci bénéficie d'une assistance soutenue et récurrente dans ses activités d'apprentissage et de développement professionnel ${ }^{1}$. Dans cette optique, le portfolio est aussi conçu comme un instrument facilitant le feedback et la supervision différée. En ce sens, elle confere au portfolio une place privilégiée dans la démarche d'évaluation formative, enchâssée au sein des activités d'enseignement et d'apprentissage. Ainsi, le rapport Tomorrow's doctors ${ }^{38}$ recommande explicitement que " les étudiants doivent recevoir une information régulière et conséquente concernant leur développement et leurs progrès ». Il précise que « les journaux de bord et les portfolios personnels, qui permettent aux étudiants d'identifier leurs forces et leurs faiblesses et d'orienter adéquatement leurs apprentissages, peuvent servir de support à une telle information ». Il souligne encore que "leur usage permet de mettre en exergue l'importance d'entretenir un portfolio, qui deviendra nécessaire une fois qu'ils seront diplômés dans l'optique de leur recertification périodique ».

\section{Caractéristiques des porffolios}

Nous envisagerons successivement les aspects liés aux contenus, aux types et aux supports possibles d'un portfolio.

\section{Le contenu d'un portfolio}

On a déjà souligné que le format et le contenu d'un portfolio peuvent considérablement varier en fonction des contextes d'utilisation et des finalités qui lui sont assignées. Dès lors que l'on admet qu'il doit être davantage qu'un simple inventaire ou qu'un simple carnet de bord et qu'il doit comporter les trois composantes déjà évoquées de support à l'apprentissage, à l'évaluation et à la démarche réflexive, on s'accorde généralement pour considérer que doivent figurer dans un portfolio ${ }^{1,2}:$ a) une compilation de documents variés : récits de situations cliniques, description de problèmes de santé auxquels l'étudiant a été confronté, notes d'analyse bibliographique critique, description de projets en cours, vidéoclips d'entretiens avec des patients ou de tâches procédurales, etc. ; b) une synthèse articulée et « réflexive » des apprentissages développés à partir de ce matériau.

\section{Une compilation de documents variés}

Il n'y a pas de restriction a priori quant à la nature des documents archivés dans un portfolio. Ceux mentionnés cidessous ne sont que des exemples couramment rencontrés ${ }^{2}$ : - des récits de situations cliniques auxquelles l'étudiant a été confronté. Il peut s'agir de situations rapportées de façon relativement extensive, faisant référence à un problème de santé considéré dans sa globalité. Il peut s’agir aussi de situations rapportées de façon plus restreinte, faisant référence à ce que la littérature désigne souvent sous le terme d'évènement critique (critical incident), étant précisé qu'il ne s'agit pas nécessairement d'une situation clinique difficile ou au pronostic péjoratif et que l'on devrait plutôt parler d'événement significatif. Dans tous les cas, les informations documentées doivent permettre d'aborder plusieurs aspects de l'action médicale (diagnostic bioclinique, relation médecin-patient, éducation thérapeutique, aspects déontologiques et éthiques, etc.) ;

- des comptes rendus de diverses activités plus ou moins formelles auxquelles a participé l'étudiant dans le cadre de son exercice professionnel : réunions de service, confrontations anatomo-cliniques, séances de bibliographie, exposés, conférences, etc.

- des comptes rendus de lecture de toute forme d'information médicale (articles, livres, documentation électronique, documents d'information ou de promotion délivrés par l'industrie pharmaceutique, etc.) ;

- des comptes rendus d'entretiens formels ou informels avec diverses personnes-ressources: pairs, maîtres de stage, tuteurs ou mentors, etc. ;

- des enregistrements audio/video d'entretiens avec des patients ou leurs familles ou de tâches procédurales (gestes techniques);

- des journaux de bord : relevés plus ou moins systématisés d'activités accomplies, effectués selon une périodicité variable (quotidienne, hebdomadaire, mensuelle, etc.).

\section{Une synthèse articulée des apprentissages développés à partir de ce matériau}

Il s'agit de montrer comment les activités professionnelles sélectionnées par l'étudiant ont été exploitées à des fins d'apprentissage, de gestion et de réflexion, en établissant des liens entre les tâches et activités spécifiques accomplies, les connaissances construites et les compétences développées. Des consignes variées peuvent être formulées à l'étudiant pour l'aider dans cette démarche cognitive ${ }^{1,2,45,46}$; d'une façon générale, elles invitent l'étudiant à documenter les aspects suivants dans son portfolio.

- l'expérience : qu'est-il arrivé, qu'est-ce qui a été fait, vu, écrit, etc. ? 
- le questionnement : en quoi l'expérience vécue m’a posé problème?

- la détermination de besoins d'apprentissage : que puisje ou que dois-je apprendre à partir de cette expérience pour être en mesure de mieux la comprendre, de résoudre les problèmes posés?

- la planification des activités d'apprentissage : comment puis-je effectuer ces apprentissages? A partir de quelles ressources?

- l'évaluation des retombées de l'ensemble de la démarche: qu'ai-je appris à partir de cette expérience? Comment l'ai-je appliqué ? Quelles leçons puis-je en tirer pour ma pratique future? etc.

Le caractère rédigé des différents éléments de contenu évoqués, et notamment de cette synthèse, est considéré par plusieurs auteurs comme essentiel. Walker ${ }^{47}$ souligne à cet égard que l'écriture est bien plus qu'une activité mécanique et qu'elle peut promouvoir l'activité réflexive pour plusieurs raisons :

a) elle favorise une perception plus « objective " des expériences vécues, grâce à un processus de distanciation, en aidant l'étudiant à distinguer les faits et l'interprétation qu'il en a faite ; b) elle aide l'étudiant à appréhender la composante affective du processus d'apprentissage ; c) elle favorise la dynamique d'ancrage des nouveaux apprentissages à partir des connaissances antérieures de l'étudiant ; d) elle favorise la mise en perspective des apprentissages avec les différentes expériences successivement rencontrées ; e) elle facilite les interactions entre les étudiants, améliore la qualité de l'interaction de ceux-ci avec leurs formateurs et prépare les interactions qu'ils auront à développer avec les autres professionnels de santé.

\section{Les différents types de portfolio}

Par nature, les portfolios sont des productions individuelles. Cependant, en fonction des buts poursuivis, des consignes générales ou plus spécifiques peuvent contribuer à délimiter et à organiser le contenu d'un portfolio. Elles peuvent concerner l'étendue ou l'architecture du contenu du portfolio.

\section{L'étendue du contenu des portfolios}

D’une façon générale, il existe deux types de portfolios : les portfolios non-sélectifs (all inclusive portfolio) et les portfolios sélectifs (selection portfolio) ${ }^{11}$.

\section{Les portfolios non sélectifs}

Ils sont constitués par le recueil de tout le travail produit par un étudiant dans le cadre d'un cours, d'un stage ou d'un cycle de formation ; ils fournissent une documentation exhaustive de ses réalisations, qui rend compte de ses progrès au jour le jour. Ils renseignent de manière intéressante sur la qualité et la quantité des expériences auxquelles l'étudiant est exposé et fournissent par là même des informations irremplaçables sur la nature du curriculum. Ils réclament en revanche un temps considérable d'élaboration par les étudiants et d'analyse par les enseignants superviseurs, exposent de ce fait volontiers au risque d'abandon ou de travail plus superficiel et posent des problèmes logistiques de stockage des informations ${ }^{11}$.

\section{Les portfolios sélectifs}

Un portfolio sélectif est un travail qui répond à des objectifs spécifiques déterminés en fonction d'un but explicite. Ils peuvent être définis par les enseignants responsables de manière institutionnelle ou conjointement par les enseignants et les étudiants; dans ce cas, le cahier des charges du travail à documenter dans le portfolio et les critères d'appréciation sont connus de l'étudiant au début de la séquence de formation concernée ${ }^{11}$. Ils peuvent aussi être définis graduellement de manière contextualisée, à partir d'une démarche de pratique réflexive, ancrée sur les taches quotidiennes, ce qui implique alors une supervision périodique planifiée du travail de l'étudiant ${ }^{21,48}$.

\section{Larchitecture des portfolios}

A partir d'une étude rétrospective exploratoire de l'usage du portfolio dans le cadre de programmes de formation d'infirmières et de sages-femmes, Webb et al. ${ }^{49}$ ont proposé une typologie descriptive des portfolios, qui complète la distinction précédemment exposée entre portfolio sélectifs et non sélectifs en faisant référence à l'architecture des portfolios. De façon très imagée, ils distinguent quatre modèles :

- le modèle du " panier de courses" (shopping trolley) est une forme peu structurée de portfolio non sélectif à l'intérieur duquel l'étudiant rassemble de manière non systématisée et non exhaustive, en partie aléatoire, un peu " comme l'on fait son marché ", un certain nombre de documents utilisés ou produits au cours de ses apprentissages ; le seul critère de choix est ce que lui-même pense être approprié. L'articulation des éléments du contenu et la cohérence globale de tels portfolios sont souvent faibles ; ils sont le plus souvent réservés à un usage strictement personnel et sont rarement supervisés et exploités par un enseignant ;

- le modèle du " grille-pain " (toast rack) est une forme très structurée et très standardisée de portfolio sélectif. Des objectifs pré-déterminés, assimilés aux fentes du grille-pain, en nombre limité et souvent dérivés de recom- 
mandations curriculaires très normatives, sont à documenter systématiquement pour chaque module d'enseignement ou pour chaque séquence de stage, souvent à l'aide d'imprimés pré-formatés. La compilation finale de ces fiches permet rarement une vision intégrée des apprentissages qui ont été effectués.

- le modèle du "gâteau mixé " (cake mix) considère que le tout est supérieur à la stricte somme des parties ; les étudiants sont invités à documenter une construction intégrée de leurs différents résultats d'apprentissage, en élaborant des commentaires réflexifs critériés, c'est-à-dire argumentés par rapport à des référentiels de pratique ;

- enfin, le modèle de la " colonne vertébrale " (spinal column) propose qu'un répertoire de compétences (chacune d'entre elles étant assimilé à une vertèbre) serve de colonne vertébrale structurante à la démonstration des apprentissages des étudiants. Les différentes preuves qu'ils accumulent sont comparées aux différentes racines nerveuses; chacune d'entre-elles documente une dimension spécifique de la compétence et doit, si elle vise à accréditer une autre composante du répertoire, être replacée dans son contexte adéquat. L'ensemble du répertoire démontre à la fois une autonomie des composantes, une interdépendance et une cohérence globale. Bien que pour l'instant purement rhétorique, et même essentiellement métaphorique, cette typologie offre un cadre d'analyse de différentes pratiques développées autour du concept de portfolio. Elle souligne l'importance des consignes formulées aux étudiants pour leur travail.

Pour les auteurs, les deux premiers modèles semblent avoir des limites pour rendre compte d'une authentique démarche d'apprentissage réflexif.

\section{Les supports matériels des portfolios}

Le support matériel utilisé varie d'un simple carnet de stage (note-book) à des dispositifs électroniques plus ou moins sophistiqués (CD-Rom, ordinateurs de poche de type palm ou espaces numériques disponibles sur des serveurs et accessibles via une connexion Internet à partir d'un micro-ordinateur). Certaines expériences récentes développées en formation initiale, telles que le programme "SkillBase " à Manchester, montrent que l'on peut développer en quelques semaines avec succès des dispositifs techniques viables, à des coûts jugés raisonnables ${ }^{50}$.

Au Canada, l'ambitieux programme de formation continue pour médecins spécialistes $\mathrm{MOCOMP}^{\circledR}$ a introduit une version papier d'un portfolio d'apprentissage en 1995 et une version électronique dès 1996 ; la proportion de médecins utilisant cette dernière est d'environ deux tiers ${ }^{51}$. Plusieurs travaux suggèrent que des effets positifs pour- raient être mis au crédit du support électronique. Fung $e t$ al..$^{52}$ ont trouvé, par exemple, qu'un dispositif de portfolio électronique, proposé à des résidents de gynécologieobstétrique et leur donnant accès à des ressources directes via Internet, augmentait significativement leur perception d'une capacité à autodiriger leurs apprentissages. Plusieurs expériences en formation initiale rapportent des facilités permises par le recours à un portfolio via Internet, telles que l'accès à des avis de pairs ou d'experts ${ }^{3,54}$.

Certaines observations faites en contexte de formation médicale continue sont cependant plus contrastées, témoignant d'un intérêt plus faible à l'égard du portfolio électronique : d'une manière générale, il semble que l'acceptabilité du portfolio est accrue lorsqu'une flexibilité est offerte quant au choix du support ; l'adhésion au portfolio électronique reste largement tributaire des habitudes personnelles et de la familiarité avec l'outil informatique ${ }^{55}$.

\section{Les conditions d'implantation et d'accompagnement du portfolio}

La décision d'introduire le portfolio dans un dispositif curriculaire est tout sauf neutre. Challis dit même explicitement que "portfolio-based learning is not a soft option" ${ }^{2}$. Compte tenu des nouveaux rôles qui sont respectivement sollicités des étudiants et des enseignants, une telle démarche est exigeante pour les uns et pour les autres ; son initiation doit être préparée et son développement envisagé graduellement. En raison même des bases conceptuelles qui ont favorisé son développement, le portfolio sousentend en outre que l'on ait choisi d'infléchir le curriculum concerné d'une logique d'enseignement vers une logique d'apprentissage ${ }^{1,2,7,11}$. Cette notion est de fait implicite ; l'un des enjeux de la réussite d'un projet pédagogique centré autour du portfolio est précisément de la rendre explicite auprès de toutes les parties prenantes. Nous examinerons successivement les modalités d'implantation et de développement et nous discuterons certaines conditions spécifiques de réussite.

\section{Modalités d'implantation et de développement \\ Règles générales}

Lanalyse de la littérature ${ }^{1,2}$ conduit à recommander que des réponses soient explicitement apportées aux questions suivantes avant le début de l'expérience.

- Qui aura accès au portfolio et que deviendra le matérian élaboré? 
Compte tenu de la nature éminemment personnelle du contenu d'un portfolio, et notamment de sa composante réflexive, il convient de lui appliquer les mêmes règles de confidentialité qu'à toute information médicale. En dehors du mentor ou des évaluateurs électivement et explicitement dédiés à la supervision du travail de l'étudiant, il est recommandé qu'aucune autre personne n'ait accès au portfolio et qu'aucune exploitation de son contenu - par exemple à des fins de recherche ou d'enseignement au profit d'autres personnes - ne soit effectuée sans le consentement de l'étudiant.

- Qui peut écrire dans le portfolio?

Dans certaines expériences curriculaires, les mentors personnels de chaque étudiant intègrent leurs commentaires rétroactifs dans le portfolio, notamment au décours des entretiens formels périodiques qu'ils ont avec l'étudiant ${ }^{2,56}$. Ce point doit être clairement spécifié.

- Au profit de quel type d'évaluation le portfolio sera-t-il exploité?

Le point de savoir s'il est licite d'utiliser un portfolio à des fins d'évaluation sommative ou s'il convient de le réserver à une évaluation formative fait l'objet de questions examinées plus loin. En tout état de cause, il ne doit y avoir aucune ambiguïté à cet égard aux yeux de l'étudiant.

\section{Implantation et développement}

Limplantation d'un portfolio, par les conceptions pédagogiques qu'elle implique, constitue souvent une rupture par rapport aux pratiques antérieures des étudiants et des enseignants, qu'il ne faut pas sous-estimer. Plusieurs auteurs recommandent de démarrer un tel projet avec des objectifs réalistes, bien délimités, et de développer graduellement des objectifs de plus en plus ambitieux ${ }^{2}$. Dans une période exploratoire, on limitera par exemple la consigne de travail au recueil d'un nombre limité d'événements critiques par période de stage et à leur analyse réflexive. Pour dissiper les conceptions erronées, les malentendus, voire les craintes chez les uns et les autres, il est également recommandé d'organiser des ateliers de préparation et de formation des étudiants et des enseignants concernés. Le témoignage et la participation de pairs ayant participé à des expériences réussies peuvent s'avérer à cet égard tout à fait féconds.

\section{Conditions spécifiques de réussite}

A l'issue d'une recherche qualitative faisant appel à des entrevues semi-structurées conduites auprès de 13 enseignants mentors impliqués dans l'expérience du portfolio à la faculté de médecine de Maastricht, Driessen et al. ${ }^{56}$ ont identifié quatre catégories de conditions susceptibles de favoriser la réussite de son implantation : 1) la supervision de l'étudiant ; 2) la structure du portfolio et les recommandations formulées aux étudiants ; 3 ) les expériences et le matériel colligés dans le portfolio ; 4) le type d'évaluation développé à partir du portfolio.

\section{La supervision de l'étudiant}

Quelle que soit la dénomination employée pour désigner la personne-ressource [superviseur, mentor, formateur (trainer), moniteur (coach), etc.], la supervision de l'étudiant est tenue par la plupart des auteurs pour être essentielle dans une approche pédagogique centrée sur le portfolio ${ }^{1,2,11,44,56}$. Elle répond à la nécessité d'assister l'étudiant dans sa démarche d'auto-évaluation et d'auto-direction de ses apprentissages, condition nécessaire d'une pratique réflexive mais qui n'est pas spontanée, contrairement aux postulats de la pédagogie d'adultes ${ }^{57}$ qui sont aujourd'hui démentis ${ }^{58,59}$. Lautonomie de l'apprenant doit donc clairement être un résultat visé par l'utilisation du portfolio et non pas une caractéristique présente du seul fait qu'on l'utilise, confusion pourtant souvent faite. La qualité de la composante réflexive produite dans le portfolio est favorisée par une attitude appropriée du mentor ${ }^{60}$. Cette activité de mentorat peut revêtir des modalités plus ou moins formelles : rencontres périodiques planifiées, rencontres plus opportunistes à l'occasion d'une activité conjointe, rencontres téléphoniques ; il est possible d'y associer le cas échéant d'autres personnes : pairs ou autres professionnels de santé ${ }^{1,11}$. A la fin d'une rencontre formelle, les éléments essentiels de la rétroaction formulée ont intérêt à être consignés par écrit, par l'étudiant ou par le mentor, ainsi que les pistes d'apprentissages identifiées, de façon à préparer et mieux exploiter la rencontre suivante ${ }^{2,56}$.

\section{La structure du portfolio et les recommandations formulées aux étudiants}

Plusieurs mentors trouvent un intérêt à ce que les consignes de travail recommandent un portfolio de type structuré, au moins au début d'une expérience et pour les étudiants plus faibles, quitte à favoriser une structure plus flexible dans un second temps ${ }^{56}$. Ainsi, dans plusieurs expériences rapportées, les étudiants soulignent qu'ils n'ont pas immédiatement compris ce qu'on attendait d'eux dans leur travail d'élaboration du portfolio ${ }^{1,32,61}$. A l'inverse, formuler d'emblée des recommandations trop directives peut se révéler être un obstacle pour les étudiants qui ont déjà développé des bonnes habiletés réflexives ${ }^{56}$.

\section{Les expériences et le matériel colligés dans le portfolio \\ Pour qu'une réelle interaction puisse se développer entre}


l'étudiant et son mentor, il faut que les expériences rapportées soient suffisamment pertinentes et que le matériau élaboré à partir d'elles soit suffisamment riche, faute de quoi l'exercice devient futile et démotivant ${ }^{56}$. Pour cela, il est nécessaire que le contexte de pratique dans lequel l'étudiant est immergé soit adapté aux compétences visées. Il est également fondamental que les conditions générales de travail favorisent une élaboration du portfolio ancrée authentiquement et régulièrement dans la pratique de l'étudiant, afin d'éviter une rédaction hâtive, superficielle ou réduite à un pur exercice de style, quelques jours seulement avant la date de rendez-vous avec le mentor ${ }^{1}$.

\section{Le type d'évaluation développée à partir du portfolio}

Ce point est l'un de ceux qui fait le plus débat aujourd'hui. En s'appuyant sur les résultats de leur étude, Driessen $e t$ al. ${ }^{56}$ militent pour que l'évaluation des apprentissages documentés par le portfolio soit sommative. Les mentors interrogés craignent, dans le cas contraire, que les étudiants se désengagent d'une activité aussi exigeante si elle n'est pas prise en compte sous forme de note. L'opinion de Rees ${ }^{18}$ est convergente avec ce point de vue, en considérant en outre que l'évaluation sommative est de nature à renforcer les apprentissages nécessaires au développement d'une pratique réflexive, qui est l'un de ses buts essentiels et ce, tant en formation pré-graduée que graduée. Plusieurs expériences rapportées dans la littérature semblent accréditer ces points de vue ${ }^{41,62}$, un nombre significatif d'étudiants arrêtant de travailler sur un portfolio purement formatif, notamment si, simultanément, ils doivent préparer d'autres épreuves sommatives.

A l'inverse, plusieurs auteurs soutiennent que le fait d'utiliser un portfolio à des fins d'évaluation sommative risque de corrompre sa fonction d'outil réflexif ${ }^{1,2}$. Ils font l'hypothèse que des aspects essentiels ayant trait au développement personnel et professionnel de l'étudiant pourraient être omis ou minorés pour satisfaire des critères d'évaluation pris en compte ; le contenu serait dès lors davantage orienté vers les exigences de l'évaluateur que vers les besoins de l'apprenant. Dans une étude de 1996, Snadden et al. ${ }^{6}$ ont vérifié que lorsque l'évaluation du portfolio avait une fonction sommative, des généralistes en phase finale de formation (registrar) rapportaient significativement moins d'événements critiques ayant eu une évolution défavorable. Pourtant, associé à d'autres auteurs dans une étude concernant cette fois le cursus pré-gradué, et prenant acte que le portfolio était en mesure de fournir des informations sur des dimensions de la compétence mal prises en compte par les autres outils d'évaluation, Snadden concédait que, sous certaines conditions, le portfolio méritait d'être intégré comme partie de l'examen final sanctionnant ${ }^{63}$. D'autres exemples d'utilisation satisfaisante du portfolio à des fins à la fois formative et sommative ont été rapportés pour certains cursus de professionnels de santét ${ }^{64}$.

Cette discussion est rendue encore plus complexe quand, au-delà de l'impact d'une utilisation formative du portfolio sur la nature et sur la qualité des apprentissages, dans une perspective sommative et notamment certificative, on s'interroge sur la difficulté du portfolio à satisfaire les critères métrologiques de validité et surtout de fidélitét ${ }^{65}$. Ce point est abordé plus loin au chapitre des questions en suspens.

Quoi qu'il en soit, la plupart des auteurs s'accordent pour considérer que, tant dans une logique sommative que formative, la démarche évaluative développée autour du portfolio a avantage à s'appuyer sur une interprétation critériée du travail de l'étudiant ${ }^{1,2,11}$. Un répertoire explicite des compétences visées, articulé autour d'une gamme de tâches professionnelles clairement identifiées, les unes et les autres faisant l'objet de descripteurs qualitatifs hiérarchisés en niveaux de performances (rubrics) ${ }^{11}$, facilite le travail d'auto-évaluation de l'étudiant et améliore la qualité de la supervision et de la rétroaction, en rendant visibles à l'étudiant les critères de jugement. Des modèles didactiques de la compétence médicale tels que ceux développés par Harden et al. ${ }^{35,36,41}$ peuvent clairement faciliter l'élaboration de tels outils.

\section{Bénéfices attendus et impacts observés, limites et difficultés, questions en suspens autour de lutilisation du porffolio}

\section{Bénéfices attendus et impacts observés}

Les bases conceptuelles sous-jacentes à l'utilisation du portfolio en éducation des sciences de la santé présentent un fort degré de crédibilité. Elles sont cohérentes avec les plus récentes données issues de la recherche dans les domaines des sciences de l'apprentissage ${ }^{66}$, de l'enseignement contextualisé et stratégique ${ }^{67}$, de l'expertise professionnelle ${ }^{27}$ et de l'évaluation authentique ${ }^{8}$.

Les bénéfices attendus de l'utilisation du portfolio dans les programmes de formation des professionnels de santé sont de ce fait légitimement ambitieux ${ }^{1,2,11}$. Le développement d'une autonomie d'apprentissage chez l'étudiant et le futur professionnel, qui sous entend l'acquisition d'une capacité d'auto-évaluation et d'autodirection entretenue tout au long de la carrière profes- 
sionnelle (self-directed learning et life long learning), est l'une des finalités les plus explicites de l'utilisation du portfolio ${ }^{1,2,44}$. L'acquisition d'une capacité réflexive à l'égard de son développement professionnel et de ses pratiques professionnelles est la seconde finalité majeure, en lien avec la précédente. L'une et l'autre supposent le développement de stratégies métacognitives à un haut degré, qui permettront au professionnel de construire et d'exploiter en action le savoir tacite qui est l'une des marques de l'expertise ${ }^{25,27}$. L'une et l'autre répondent aussi aux exigences liées au mandat social du professionnel de santé, qui fondent les valeurs du professionnalisme ${ }^{68,69}$.

Les tableaux 1 et 2 résument respectivement les bénéfices qui ont été rapportés pour les étudiants et pour les enseignants dans le cadre de l'une ou l'autre des multiples expériences documentées dans la littérature ${ }^{1,2,11,70}$. Spécifiquement, certains résultats concernant des expériences conduites en cursus pré-gradué montrent, par exemple : que des étudiants ayant utilisé un portfolio ont obtenu de meilleurs scores lors de l'évaluation de leurs apprentissages en oncologie à l'aide d'examens cliniques objectifs structurés (ECOS) ${ }^{62}$; que l'utilisation soutenue du portfolio, mesurée par la quantité de texte écrit rapportée, était corrélée significativement au succès à l'examen final $^{71}$; que les étudiants ayant développé une vision positive de l'utilisation du portfolio cotent de façon plus élevée les apprentissages qu'ils ont effectué dans le domaine des habiletés de communication ${ }^{72}$; que des étudiants ayant utilisé précocement un portfolio dans leur programme jugent extrêmement positivement l'aide que cela leur a apporté en termes de métacognition et de développement personnel et professionnel ${ }^{12}$; qu'environ deux tiers des étudiants d'une promotion ayant utilisé un portfolio pendant leur externat considéraient qu'il constituait une aide significative pour améliorer leurs habiletés de communication, réviser leur travail et apprendre de façon autonome à partir des problèmes de santé rencontrés ${ }^{73}$. Dans le cadre de la formation graduée en médecine générale, on a montré que l'utilisation du portfolio, à condition d'être supervisée de façon adéquate, augmentait la confiance des résidents en eux-mêmes lors des premiers mois de pratique en milieu ambulatoire après avoir quitté l'hôpital, améliorait l'adéquation des apprentissages développés par rapport aux compétences visées et facilitait l'exploitation formative des situations difficiles rencontrées par les étudiants ${ }^{44}$. Dans les milieux où le portfolio est utilisé comme support de la formation médicale continue du généraliste, les médecins soulignent l'effort supplémentaire requis par rapport à d'autres méthodes plus conventionnelles mais aussi les retombées nettement plus importantes en termes de formation ${ }^{1,22,74}$.

\section{Difficultés et limites}

Elles concernent la vulnérabilité du dispositif et l'incertitude quant aux retombées effectives, notamment à long terme.

\section{La vulnérabilité de l'approche pédagogique}

En raison même des finalités poursuivies, qui sont ambitieuses, la tenue d'un portfolio réflexif est exigeante pour les étudiants et les enseignants concernés. De ce fait, un dispositif d'enseignement, d'apprentissage et d'évaluation centré autour de l'utilisation du portfolio est relativement vulnérable.

Plusieurs résultats convergents rapportés dans la littérature accréditent cette notion. Ainsi, même quand ils en perçoivent fortement l'utilité, une proportion importante d'étudiants concernés, jusqu'à $93 \%$ d'une promotion dans certaines expériences ${ }^{73}$, trouvent que l'obligation d'avoir à écrire dans leur portfolio est stressante. Un certain nombre considèrent qu'ils sont capables de développer mentalement une authentique démarche réflexive, que l'écriture n'est pas indispensable à cet effet et qu'elle constitue dès lors une perte de temps ${ }^{2,62}$. Il n'est pas exceptionnel que les étudiants ne consignent les événements dans leur portfolio que dans les jours qui précèdent leur rencontre avec leur mentor ou à la toute fin du stage, parfois très à distance de la date où les expériences ont été vécues ${ }^{1,49,73}$. Dans une étude anglaise, parmi 92 résidents en fin de formation en médecine générale, seuls $65 \%$ colligeaient l'information de façon régulière ${ }^{60}$. Dans une autre étude britannique, l'utilisation du portfolio au sein d'une population comparable décroissait au bout de six mois ${ }^{44}$. La perception des étudiants à l'égard de la démarche est identifiée comme très variable ${ }^{21,61,63,72}$ : à coté de visions nettement positives, d'autres sont globalement neutres voire franchement défavorables.

Les obstacles et les menaces qui hypothèquent la viabilité pédagogique du portfolio, telles qu'elles sont identifiées par des recherches qualitatives, sont remarquablement similaires d'une étude à l'autre ${ }^{1,2,6,10,60}$. Ils concernent : a) le manque de temps, notamment lorsque les tâches qui entrent en concurrence sont des activités exigeantes comme les soins mais aussi lorsqu'il s'agit d'autres activités académiques (préparation d'examens, de mémoires, de thèses, etc.) ; b) l'absence, l'insuffisance pédagogique ou la mauvaise qualité relationnelle de la supervision, qu'il s'agisse du processus de mentorat autour du portfolio ou du feedback par les personnes ressources dans les différents milieux de pratique des étudiants ; c) le manque de clarté des consignes de travail quant aux contenus et aux formats attendus pour le 


\section{Tableau 1 : \\ Bénéfices rapportés de l'utilisation du portfolio pour l'étudiant}

- Développement d'une vision positive d'eux-mêmes en tant qu'apprenants

- Prise de conscience de la qualité de leurs apprentissages (étendue, profondeur) et des stratégies mises en œuvre

- Facilitation des apprentissages signifiants et approfondis (meaningful and deep learning)

- Facilitation du transfert des apprentissages

- Meilleure appréciation des objectifs de formation professionnelle définis par l'institution

- Meilleure exploitation du feedback reçu de la part des différentes personnes ressources

- Prise de conscience du rôle positif de l'écriture dans l'apprentissage

- Amélioration de la confiance en soi et en ses apprentissages

- Meilleure compréhension des exigences de l'apprentissage comme processus continu durant toute la vie (lifelong learning)

- Amélioration des interactions de l'étudiant avec ses pairs et avec ses différentes personnes ressources

Tableau 2 :

Bénéfices rapportés de l'utilisation du portfolio pour les enseignants

- Implication accrue dans le processus d'évaluation du programme

- Meilleure connaissance des exigences réelles, des faiblesses et des forces du curriculum

- Amélioration de la qualité de ses interactions avec les étudiants

- Prise de conscience accrue des besoins, forces et faiblesses des étudiants

- Amélioration de la collégialité avec les enseignants de la même discipline et des autres disciplines

- Prise de conscience de la possibilité et des retombées positives d'une sollicitation d'un support institutionnel pour améliorer son enseignement

- Identification de pistes de recherche en pédagogie 
portfolio ; d) le caractère non explicite des critères pris en compte pour l'évaluation du portfolio.

\section{Des retombées effectives encore incomplètement documentées}

Nous avons rapporté précédemment un ensemble de résultats convergents qui accréditent la notion d'un impact positif du portfolio en tant qu'aide au développement des capacités d'apprentissage autonome et au développement d'aptitudes réflexives.

Plusieurs incertitudes demeurent cependant tant à la valeur ajoutée propre du portfolio à l'égard de l'apprentissage. La planification d'apprentissages de nature réflexive à partir des expériences colligées dans un portfolio ne convient pas à tous les styles d'apprentissages ${ }^{2}$. Plusieurs travaux confirment que les expériences rapportées sont parfois, pour une partie des étudiants, purement descriptives $^{75}$. La composante réflexive du portfolio est parfois faible : dans une étude déjà citée ${ }^{60}$, seuls $42 \%$ de résidents en médecine générale exploitaient leur portfolio au profit d'une démarche d'apprentissage réflexif ${ }^{60}$. Parmi ceux qui produisaient des portfolios non réflexifs, plusieurs avaient pourtant une pratique professionnelle authentiquement réflexive comme l'attestaient des informations obtenues à partir d'autres sources ; dans ce cas, c'est la pertinence opérationnelle de l'outil pour en rendre compte qui semble en cause. Dans un très récent commentaire éditorial critique $^{76}$, Albanese suggère que la tenue d'un journal pourrait ne pas être une méthode optimale pour promouvoir l'apprentissage réflexif, en évoquant qu'il puisse aider certains étudiants mais qu'il puisse aussi être un obstacle pour d'autres. Les futures recherches devront donc s'attacher à apporter des réponses à un certain nombre de questions non résolues ${ }^{77}$ : quelles sont les conditions à satisfaire pour que l'implantation du portfolio ait aussi régulièrement que possible des impacts conformes à ses intentions ? Quelle est la part des impacts respectivement imputables à la tenue du portfolio par l'étudiant, aux interactions induites avec les enseignants dans le cadre du processus de mentorat et d'évaluation formative ou encore aux orientations spécifiques du programme favorisées en tant que telles par l'introduction du portfolio ? Les retombées concernant l'aide du portfolio au développement d'une pratique authentiquement réflexive se maintiennent-elles sur le long terme?

\section{Une question épineuse en suspens: l'utilisation du portfolio en tant que support d'évaluation sommative}

Nous avons déjà rapporté le point de vue des auteurs qui soutiennent que le fait d'utiliser un portfolio à des fins d'évaluation sommative risque de corrompre sa fonction d'outil réflexif ${ }^{1,2}$. Une autre question majeure concerne le point de savoir si le portfolio offre des garanties de rigueur suffisantes ${ }^{20}$ pour étayer des décisions sommatives aux enjeux importants, notamment de certification voire de recertification. Par rapport à un tel objectif, la perspective docimologique de l'évaluation, qui s'adosse à une théorie de la mesure, requiert que les outils utilisés aient un haut degré de validité et de fidélité. Ceci conduit généralement à privilégier les outils caractérisés par une forte standardisation, avec le souci d'atteindre une forte objectivité, en réduisant la part de l'interprétation "subjective " dans le jugement évaluatif. Par nature, le portfolio est une construction personnelle peu standardisée, qui cherche à promouvoir une pensée réflexive, c'est-à-dire une démarche à la fois constructiviste et phénoménologique, où la part de l'interprétation et du jugement de valeur à l'égard de ses pratiques est primordiale. Il n'est dès lors guère étonnant que les études visant à vérifier les garanties métrologiques du portfolio aient mis en évidence des problèmes quant à ses qualités psychométriques $^{63,78,79}$. Les réserves les plus sérieuses concernent la fidélité : la concordance inter-correcteurs, (faculté de deux correcteurs à coter le portfolio avec le même score) a été trouvée très médiocre et la concordance intra-correcteur - ou consistance - (faculté d'un correcteur à coter le portfolio avec le même score lorsqu'il l'évalue à deux moments différents) seulement moyenne. Au nom de l'orthodoxie docimologique, Roberts et al. ${ }^{20}$ dénient de ce fait au portfolio toute légitimité à servir de support à une évaluation sommative, en indiquant même qu'une telle utilisation conduirait à revenir " aux jours sombres du passé ", lorsque l'on se contentait d'une simple validité apparente des moyens d'évaluation. Ce point de vue n'est cependant pas partagé par tous les auteurs. Snadden ${ }^{80}$ considère que vouloir améliorer à tout prix les qualités métrologiques de l'évaluation d'un portfolio revient à vouloir mesurer ce qui n'est pas mesurable et, dès lors, à privilégier ce qui est facile à mesurer plutôt que ce qu'il est pertinent d'évaluer. Driessen et al. ${ }^{13}$ partagent ce point de vue. Ils soulignent les limites et le caractère réducteur de deux stratégies utilisables pour améliorer la concordance inter-correcteurs : la standardisation du portfolio, grâce à une forte structuration de son contenu et à une limitation des sources d'information acceptables dans un portfolio, d'une part ; l'entraînement des correcteurs et la structuration de leur processus de cotation à l'aide de listes de vérification (checklists) faisant référence à des critères stricts, d'autre part. Ils considèrent que l'accroissement ainsi obtenu de la fidélité se ferait au détriment de la validité du portfolio qui, par construction, vise à 
documenter des expériences personnelles, dont la puissance éducationnelle est liée au fait qu'elles se rapportent à des tâches professionnelles authentiques. Ils considèrent qu'une standardisation excessive serait antinomique avec le but poursuivi. Ces analyses sont en accord avec les réflexions actuelles qui cherchent à refonder l'évaluation des apprentissages lorsque l'on inscrit un dispositif pédagogique dans le paradigme constructiviste. Tardif ${ }^{8}$ précise ainsi que, dans cette perspective, la concordance intra-correcteur est un critère secondaire voire peu pertinent et que, dès lors que l'on se préoccupe des compétences développées par les étudiants, le respect de la validité de construit - qui concerne la justesse et la pertinence avec lesquelles l'outil renseigne sur différentes composantes du " construit » théorique des compétences visées- est essentiel ; ceci exige qu’elles soient évaluées à partir de tâches complètes, complexes et signifiantes ce qui est le cas avec le portfolio. Il insiste aussi sur la nécessité que les modalités d'évaluation rendent compte des métamorphoses cognitives et métacognitives de l'étudiant et sur l'importance qu'elles répondent à une validité « écologique ", qui suppose une similitude entre les conditions d'évaluation et le contexte prévisible de mobilisation ultérieure des connaissances et des compétences construites. Van der Vleuten $e t ~ a l .{ }^{8 I}$ indiquent que l'on fait fausse route en considérant que fidélité et validité sont des caractéristiques intrinsèques à l'outil utilisé. Ils rappellent que le facteur majeur qui altère la fidélité d'une évaluation est la non prise en compte de la spécificité de contenu - ou de domaine des situations d'évaluation et que, dès lors que l'échantillon des contenus et domaines explorés par les situations d'évaluation est suffisamment varié, le critère de fidélité peut être satisfait, même avec des méthodes relativement "subjectives ", qui font fortement appel à l'interprétation et au jugement professionnel, et non strictement standardisées. Concernant le critère de validité, ils soulignent que les conditions les plus importantes sont l'authenticité des situations d'évaluation et l'intégration des compétences. Ils plaident de ce fait pour que l'évaluation des compétences professionnelles s'appuie sur des informations émanant de sources multiples, de nature à la fois quantitative et qualitative. Ceci n'autorise pas pour autant les enseignants impliqués dans une démarche évaluative de nature sommative à saffranchir du souci de rigueur sous prétexte qu'ils exploiteraient des données qualitatives; il convient en revanche qu'ils s'approprient dans ce cas les critères de rigueur spécifique d'une telle approche, tels qu'ils ont été codifiés en recherche qualitative ${ }^{82}$. Le critère de crédibilité est homologue de celui de validité et peut être satisfait, par exemple, au prix d'une triangulation (des informations issues de sources différentes permettent des interprétations convergentes) et d'un recueil d'informations issu d'un engagement pro- longé. Le critère de fiabilité, qui répond à celui de fidélité, peut être satisfait au prix d'une traçabilité du processus d'évaluation, permettant le cas échéant une vérification externe, et en s'inscrivant dans une démarche qualité. En opérationnalisant ces principes dans une étude exploratoire concernant l'évaluation sommative de 233 portfolios, Driessen et al. ${ }^{13}$ ont montré la viabilité d'une telle approche : pendant la première année du cursus prégradué à la faculté de médecine de Maastricht, les étudiants ont discuté leur progression, documentée dans un portfolio, à l'occasion d'au moins deux rencontres avec leur mentor. A la fin de l'année, il n'y a eu discordance entre le jugement des étudiants et celui des mentors (ajournement, passage, passage avec distinction) que dans $3 \%$ des cas ; seuls $4 \%$ des portfolios ont dû être examinés par un comité plénier en raison d'une discordance du jugement de deux correcteurs indépendants; l'ensemble de la procédure a pu être mené à son terme dans un temps jugé relativement court de 42 heures, soit 11 minutes par portfolio, la réunion du comité plénier ayant duré une heure. Le débat concernant l'utilisation du portfolio à des fins d'évaluation sommative n'est certainement pas clos. Pour autant, de telles expériences montrent que des alternatives à une approche strictement docimologique de l'évaluation peuvent être développées autour d'un outil tel que le portfolio, en cherchant à concilier rigueur d'un côté et cohérence des pratiques évaluatives avec les intentions du dispositif pédagogique de l'autre.

\section{Conclusion}

Les orientations pédagogiques qui sous-tendent l'utilisation du portfolio en éducation des sciences de la santé ont une forte crédibilité conceptuelle. Les activités développées autour du portfolio offrent aux étudiants l'opportunité de solliciter fortement leurs connaissances antérieures, de s'engager activement et de façon autonome dans des stratégies d'apprentissage dans des contextes signifiants et motivants et de construire des compétences à partir de tâches professionnelles authentiques. Elles offrent à l'enseignant un rôle de motivateur, d'entraîneur, mettent l'accent sur son rôle de pourvoyeur de rétroaction, tout en exploitant ses rôles traditionnels d'expert de contenu et de modèle de rôle. Elles sont fondamentalement centrées sur le développement professionnel et la métacognition. Enfin elles s'inscrivent dans la perspective d'une évaluation des performances enchâssée aux activités d'enseignement et d'apprentissage.

Même si des résultats concernant les impacts spécifiques doivent encore être documentés, plusieurs études convergentes montrent qu'un certain nombre de ces promesses 
peuvent être tenues, au bénéfice d'un haut degré de satisfaction des étudiants et des enseignants, mais que le dispositif est assez vulnérable, menacé par de nombreuses conditions défavorables qui peuvent conduire à des désillusions. Le portfolio est une approche pédagogique exigeante, qui nécessite que l'ensemble du curriculum soit planifié, implanté et accompagné avec une grande cohérence pédagogique. Parmi les conditions nécessaires, la mise en ouvre d'une supervision de l'étudiant grâce à un mentorat compétent et périodique est sans doute l'une des plus importantes. Les enseignants qui projettent d'introduire le portfolio comme l'un des outils majeurs d'apprentissage, d'enseignement et d'évaluation dans le cadre d'un curriculum dédié à la formation de professionnels de santé doivent prendre le plus grand soin de vérifier qu'ils seront capables de créer un environnement réunissant les conditions favorables pour que l'expérience ait des chances élevées de réussite ${ }^{56}$.

\section{Contributions}

Nicole Naccache a rédigé la version initiale du manuscrit. Louise Samson et Jean Jouquan ont contribué à ses révisions successives. Les trois auteurs ont validé collégialement et solidairement la rédaction finale.

\section{Références}

1. Challis M. AMEE Medical Education Guide No. 11 (revised): Portfolio-based learning and assessment in medical education. Med Teach 1999; 21:370-86.

2. Snadden D, Thomas ML. The use of portfolio learning in medical education. Med Teach 1998;20:192-9.

3. Arter J, Spandel V. NCME instructional module: Using portfolio of student work in instruction and assesment. Eductional Measurement : issues and practice 1992;11:3644.

4. Oechsle L, Volden C, Lambeth S. Portfolio and RNs : An evaluation. J Nurs Educ 1990;29:54-9.

5. Mitchell M.The views of students and teachers on the use of portfolios as a learning and assessment tool in midwifery education. Nurse Education today 1994 ;14:38-43.

6. Snadden D, Thomas ML, Griffin EM, Hudson H. Portfolio-based learning and general practice vocational training. Med Educ 1996;30:148-52.

7. Jouquan J, Bail Ph. A quoi sengage t-on en basculant du paradigme d'enseignement vers le paradigme d'apprentissage? Exemple d'une revision conduite en résidanat de medicine générale. Pédagogie Médicale 2003;4:163-75.

8. Tardif J. Lévaluation dans le paradigme constructiviste. In: Hivon R. (Dir.) l'évaluation des apprentissages. Réflexions, nouvelles tendances et formation. Sherbrooke: Editions du CRP (Faculté d'éducation, Université de Sherbrooke), 1993:27-56.
9. Webb C, Gray M, Jasper M, McCullan M, Scholes. Models of portfolios. Med Educ 2002;36:897-8.

10. Jensen GM, Saylor C. Portfolios and professional developement in health professions. Evaluation and the Health Professions, $1994 ; 17: 344-57$.

11. Huba ME, Freed JE. Using portfolios to promote, support and evaluate learning. In : Huba ME, Freed $J E$. Learner-centered assessment on colleges campuses. Shifting the forcus from teaching to learning. Needhal Heights (MA):Allyn \& Bacon, 2000:23368.

12. Gordon J. Assessing students' personal and professional development using portfolios and interviews. Med Educ 2003;37:335-40.

13. Driessen E, van der Vleuten C, Schuwirth, van Tarwijk J, Vermunt J. The use of qualitative research criteria for portfolio assessment as an alternative to reliability evaluation: a case study. Med Educ 2005;39:214-20.

14. Legendre R. Dictionnaire actuel de l'éducation. Montréal (QC):Guérin, Paris:Eska (2e édition), 1993.

15. Scallon G. Le portfolio : un outil pour stimuler l'autoévaluation. In: Scallon G. L'évaluation des apprentissages dans une approche par compétences. Bruxelles: De Boeck Université, 2004 :285-318. 
16. Forgette-Giroux $R$, Simon M. Lapplication du dossier d'apprentissage à l'université. Mesure et évaluation en éducation $1998 ; 20: 85-103$.

17. Cole G. The definition of 'portfolio'. Med Educ 2005;39:1141.

18. Rees $C$. The use (and abuse) of the term 'portfolio'. Med Educ 2005;39:436-7.

19. Rees C. 'Portfolio' definitions: do we need a wider debate? Med Educ 2005;39:1142.

20. Roberts C. Newble DI, O'Rourke A. Portfolio-based assessment in medical education: are they valid and reliable for summative purposes. Med Educ 2002;36:899-900.

21. Mathers NJ, Challis MC, Howe AC, Field NJ. Portfolios in continuing medical education-effective and efficient? Med Educ 1999;33:521-30.

22. Dewey J. How We Think. Second revised edition. Lexington (MA) : DC Heath, 1933.

23. Garrison DR. Critical thinking and adult education: a conceptual model for developing critical thinking in adult learners. Int J Lifelong Educ 1991;10:287-303.

24. Schön D. The reflective practitioner: How professionals think in action. London: Basic Books, 1987.

25. Schön D. Educating the reflective practitioner : Toward a new design for teaching and learning in the professions. San Francisco (CA): Jossey-Bass, 1993.

26. Regher G, Norman GR Issues in cognitive psychology: implications for professional education. Acad Med 1996;71:988-1001.

27. Ericsson KA. Deliberate practice and the acquisition and maintenance of expert performance in medicine and related domains. Acad Med 2004;79 (Suppl. 10):S70-S81.

28. Mamede S, Schmidt HG. The structure of reflective practice in medicine. Med Educ 2004;38:1302-8.
29. Whittock T. Reflexive teaching, Reflexive learning. Teaching in Higher Education 1997;2:93-102.

30. King PM, Kitchener KS. Developing reflective judgment: Understanding and promoting intellectual growth and critical thinking in adolescents and adults. San franscisco (CA) : Jossey-Bass, 1994

31. Jensen GM, Saylor C. Portfolio and the professional development in the health professions. Evaluation and the Health Professions 1994;17:344-57.

32. Parboosingh J. Learning Portfolios: Potential to Assist Health Professionals with Self-Directed Learning. J. Cont. Educ. Health Prof., 1996, 16, 75-81.

33. Swick Herbert M. Toward a Normative Definition of Medical Professionalism. Acad Med, 2000;25:612-6.

34. Leung W-C. Competency based medical training: review. BMJ 2002;325:693-6.

35. Harden R. AMEE Guide No. 14: Outcome-based education: Part 1-An introduction to outcome-based education. Med Teach 1999;21:7-14.

36. Friedman Ben-David M. AMEE Guide No. 14: Outcome-based education: Part 3 -Assessment in outcome-based education. Med Teach 1999;21:23-5.

37. Shumway JM, Harden RM. AMEE Guide No. 25: The assessment of learning outcomes for the competent and reflective physician. Med Teach 2003;25:569-84.

38. General Medical Council. Tomorrow's doctors: Recommandations on Undergraduate Medical Education. London: GMC, 2003.

39. Frank JR. (Ed). The CanMEDS 2005 physician competency framework. Better standards. Better physicians. Better care. Ottawa: The Royal College of Physicians and Surgeons of Canada, 2005.

40. Frank JR, Jabbour M, Tugwell P, et al. Skills for the new millenium: report of the societal needs working group, CanMEDS 2000 Project. Annals Royal College of Physicians and Surgeons of Canada 1996;29:206-16. 
41. Harden R.M, Crosby J.R, Davis M.H, Friedman M. AMEE Guide No. 14: Outcome-based education: Part 5 -From competency to meta-competency: a model for the specification of learning outcomes. Med Teach 1999;21:546-52.

42. Glen S, Hight NF. Portfolio : an 'affective' assessment strategy? Nurse Education Today 10092;12 :416-22.

43. Snadden D, Thomas ML. Portfolio learning in general practice vocational training- does it work? Med Educ 1988;32:401-6.

44. Spencer JA, Jordan RK. Learner centred approaches in medical education. BMJ 1999;318:1280-3.

45. Redman W. Portfolios for development. London: Kogan Page, 1994.

46. Raw J, Bridgen D, Gupta R. Reflective diaries in medical practice. Reflective Practice 2005;6:165-9.

47. Walker D. Writing in reflection. In: Boud D, Keogh $R$, Walker $D$ (Eds.). Reflection: turning experience into learning. London: Logan, 1985: 52-68.

48. Grant J. Learning needs assessment: assessing the need. BMJ, 2002;324:56-9.

49. Webb C, Endacott R, Gray M, Jasper M, Miller C, Mc Mullan $M$ et al. Models of portfolios. Med Educ 2002;36:897-8.

50. Dornan T, LeeC, Stopford A, Hosie L, Maredia N, Rector A. Rapid application design of an electronic clinical skills portfolio for undergraduate medical students. Comput Methods Programs Biomed 2005;78:25-33.

51. Campbell C, Parboosingh J, Gondocz T, Babitskaya G, Pham B. Study of the factors influencing the stimulus to learning recorded by physicians keeping a learning portfolio. J Contin Educ Health Prof 2001 ; 19 :16-24.

52. Fung MFK, Walker M, Fung KFK, Temple L, Lajoie F, Bellemare $G$ et al. An internet-based learning portfolio in resident education: the KOALA multicentre programme. MedEduc, 2000;34: 474-9.
53. Supiano M.A, Fantone J.C, Grum C, Arbor A. A Web-based Geriatics Portfolio to Document Medical Students' Learning Outcomes. AcadMed 2002; 77:937-8.

54. Dornan T, Lee C, Stofford A. Skills Base: A Webbased Electronic Learning Portfolio for Clinical Skills. Acad Med 2001;76:542-3.

55. Dornan T, Carroll C, Parboosingh J. An electronic learning portfolio for reflective continuing professional development. Med Educ 2002;36:767-9.

56. Driessen EW, Van Tartwijk J, Overeem K, Vermunt JD, Van der Vleuten CPM. Conditions for successful reflective use of portfolios in undergraduate medical education. Med Educ 2005;39:1230-5.

57. Knowles M. The adult learner: a neglected species. Houston (TX): Gulf Publishing, 1973.

58. Norman G. The adult learner : a mythical species.Acad Med 1999;74:886-9.

59. Gordon MJ. A review of the validity and accuracy ofselfassesments in health professions training. Acad Med 1991;66:762-9.

60. Pearson DJ, Heywood P. Portfolio use in general practice vocational training: a survey of GP registrars. Med Educ 2004;38;87-95.

61. Bail Ph, Le Reste J-Y, Boiteux F. Le portfolio. Expérience du département de médecine générale de la faculté de Brest. La Revue du Praticien-Médecine Générale $2004 ; 18: 445-7$.

62. Finlay IG, Maughan TS, Webster DJT. A randomised controlled study of portfolio learning in undergraduate cancer education. Educational Assessment 1997;4:26596.

63. Davis MH, Friedman Ben-David M, Harden RM, Howie P, Ker J, McGhee C, Pippard MJ, Snadden D. Portfolio assessment in medical students' final examinations. Med Teach 2001;23:357-66. 
64. Jasper MA. The potential of the professional portfolio for nursing. J Clin Nursing 1995;4:249-55.

65. Roberts C, Newble DI, O'Rourke AJ. Portfolio-based assessments in medical education: are they valid and reliable for summative purposes? Med Educ 2002;36:899-900.

66. National Research Council. Committee on Learning Research and Educational Practice. Committee on Developments in the Science of Learning. Bransford $J D$, Brown AL, Cocking RR (Eds.). How People Learn. Brain, Mind, Experience and School. Washington (DC):National Academy Press, 2000.

67. TardifJ. Pour un enseignement stratégique. L'apport de la psychologie cognitive. Montréal (QC): Editions Logiques, 1992.

68. Le professionnalisme médical pour le nouveau millénaire: une charte pour les praticiens. Projet pour le professionnalisme médical (Medical Professionalism Project) développé par l'American Board of Internal Medicine (ABIM) Foundation, l'American College of Physicians-American Society of Internal Medicine (ACP-ASIM) Foundation et l'European Federation of Internal Medicine (EFIM) Pédagogie Médicale 2004;5:43-5.

69. Harris IB. New expectations for professional competence. In: Curry L,. Wergin JF et al. (Dir.). Educating professionnals. San Francisco (CA): Jossey-Bass 1993: 1752.

70. Shumway JM, Harden RM. AMEE Guide $N^{\circ} 25$ : the assessment of learning outcomes for the competent and reflective physician. Med Teach 2003;25:56984 .

71. Lonka K, Slotte V, Halttunen M, Kurki T, Tiitinen A, Vaara L, Paavonen J. Portfolios as a learning tool in obstetrics and gynaecology undergraduate training. Med Educ 2001;35:1125-30.
72. Rees $C$, Sheard C. Undergraduate medical student's views about a reflective portfolio assessment of their communication skills learning. Med Educ 2004;38:125-8.

73. Elango S, Jutti RC, Lee KL. Portfolio as learning tool: students' perspective. Ann Acad Med Singapore 2005;34:511-4.

74. Challis M, Mathers N.J, Howe A.C, Field N.J. Portfolio-based learning: continuing medical education for general practitioners - a mid-point evaluation. Med Educ 1997; 31: 22-6.

75. Routledge J, Willson M, McArthur M, Richardson B, Stephenson R. Reflection on the development of a reflective assessment. Med Teach 1997;19:122-8.

76. Albanese M. Crafting the reflective lifelong learner: why, what and how. Med Educ 2006;40:288-90.

77. Grant A, Kinnersley P, Metcalf E, Pill R, Houston $H$. Student's views of reflective learning techniques: an efficacy at a UK medical school; Med Educ 2006;40:37988.

78. Pitts J, Coles C, Thomas P. Educational portfolios in the assessment of general practice trainers: reliability of assessors. Med Educ 1999;33:515-520.

79. Pitts J, Coles C, Thomas P. Enhancing reliability in portfolio assessment: shaping the portfolio. Med Teach 2001;23:351-6.

80. Snadden D. Portfolios - attempting to measure the unmeasurable? Med Educ 1999;33:478-9.

81. Van der Vleuten CPM, Schuwirth LWT. Assessing professional competence: from methods to program. Med Educ 2005;39:309-17.

82. Lincoln YS, Guba EA (Eds.). Naturalistic Inquiry. Beverly Hills (CA): Sage, 1985.

Manuscrit reçu le 20 avril 2004 ; commentaires éditoriaux formulés aux auteurs le 16 juin 2005 et le 18 avril 2006 ; accepté pour publication le 21 avril 2006. 